\title{
Psychodermatologic disorders: case reports and review of literature
}

\author{
Beata Bergler-Czop, Ligia Brzezińska-Wcisło \\ Department of Dermatology, Silesian Medical University, Katowice, Poland \\ Head: Prof. Ligia Brzezińska-Wcisło MD, PhD
}

Postep Derm Alergol 2012; XXIX, 5: 401-406 DOI: $10.5114 /$ pdia.2012.31496

\begin{abstract}
Psychodermatologic disorders are a non-homogenous group, in which both psychological and dermatological components are present. Active co-operation between the dermatologist and the psychiatrist is necessary in all abovementioned disorders since patients first appear in dermatologic clinics. Dermatological treatment is exclusively symptomatic, since most of all, these patients require special psychiatric treatment, both pharmacological and psychotherapeutic. Here we present three cases of parasitophobia combined with factitial dermatosis and one case of self-injury in patients, in whom diagnosis of psychodermatosis was preceded by long-term and detailed diagnostic procedures for classical dermatological disorders. One should always remember about dermatitis artefacta in patients, in whom there is no positive response to treatment, and the clinical picture as well as results of additional tests comprise no logical whole picture.
\end{abstract}

Key words: psychodermatology, parasitophobia, factitial dermatosis.

\section{Introduction}

Psychodermatologic disorders are a non-homogenous group, in which both psychological and dermatological components are present $[1,2]$. Such a combination is explained by interactions between the neuroendocrine system and the immunological system, described as the "neuro-immune-cutaneous system" (NICS) [3]. Depending on the significance of the psychological or dermatological element in aetiology of the disease, Steuden et al. [4] classifies psychodermatoses into:

1) primary psychiatric disorders (with cutaneous symptoms),

2) dermatological disorders with psychogenic aetiology (developing under the influence of psychological factors, such as stress, conflict),

3) dermatological disorders, influenced by psychological factors.

Here, we are focusing on the first group of diseases.

Parasitophobia is a delusional disease. Delusions have the form of pathological thinking and erroneous beliefs, considered by patients as real ones. They are usually resistant to persuasion. The patient, most commonly a woman over 50 years of age, expresses a belief that parasites or worms infested the skin on the surface or within the skin. A typical patient brings to a doctor jars or envelopes with "parasite samples" (these are most commonly hairs, wool fragments, papers, etc.) for microscopic analysis [1-3].

The same group includes other types of delusions, such as hypochondriac delusions (fear to develop a skin cancer, venereal disease, AIDS) and phobias of bacteria and dirt, leading to compulsive hand washing and skin disinfection $[1,3,5]$.

Tactile hallucinations are disturbances of perception and consist of pathological sensory experiences without any stimuli interacting with receptors of superficial sensibility. The sensation is, most of all, a feeling of insects moving on the skin, parasites boring into the skin or current and fire action. They frequently coexist with parasitophobia [1, 3].

Body image disturbance is also a primary psychological disorder, in which the affected person is obsessively, delusionally preoccupied with the unchanged skin or has dysmorphophobia (dysmorphic disease), and he or she is excessively concerned about and preoccupied by an inex-

Address for correspondence: Beata Bergler-Czop MD, PhD, 2 A Leśna St, 42-624 Ossy, Poland, phone/fax: +48 322840877 , e-mail: bettina2@tlen.pl 
istent or small defect in the physical features. Jafferany et al. [3] described three main areas of complaints expressed by a patient from this group: face, scalp and genitals area. Body weight in women represent an additional problem, which may lead to eating disorders, such as bulimia or anorexia.

Factitial dermatitis represents an important group of disorders in dermatological practice. Factitial dermatitis (dermatitis artefacta) means that patients have injured their own skin, but they deny having intentionally inflicted the injury. Injuries usually include areas accessible to the patient's hands, but sometimes patients can use different objects to injure themselves. Injuries with neurotic background result from repetitive scratching, mostly not provoked by itching. The scratching habit becomes an uncontrollable need to remove non-existing skin changes or foreign substances present in the skin. Typical skin changes are: oozing ulcerations, erosions, scars, post-inflammatory melanoderma.

Trichotillomania belongs to impulse control disorders, in which the patient experiences the urge to pull out hair. Pulling out may be combined with twisting, breaking, cutting (trichotemnomania), and also with eating of the pulled hairs (trichophagia). The patients with trichotillomania symptoms frequently show other psychotic symptoms, including anger, depression, dementia, lowered mood, suicidal thoughts and eating disorders [3].

Active co-operation between the dermatologist and the psychiatrist is necessary in all above-mentioned disorders, since patients first appear in dermatologic clinics. Dermatological treatment is exclusively symptomatic, since most of all, these patients require special psychiatric treatment, both pharmacological and psychotherapeutic $[3,4,6]$.

Here we present three cases of parasitophobia combined with factitial dermatosis and one case of self-injury in patients, in whom diagnosis of psychodermatosis was preceded by long-term and detailed diagnostic procedures for classical dermatological disorders.

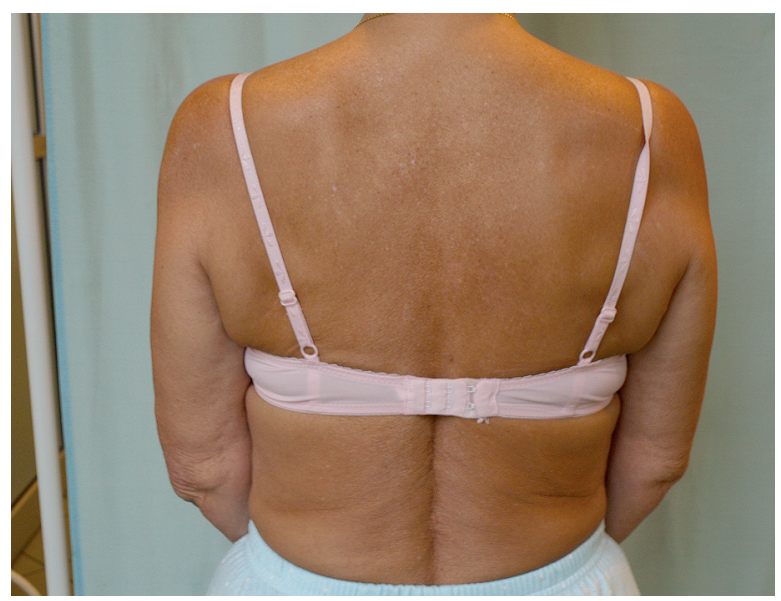

Figure 1. Patient 67 years - no skin changes

\section{Case reports}

\section{Case 1}

Patient 67 years. For about 4 years the woman was complaining about intensive skin itching, combined with the feeling of tingling and parasites moving under the skin. The beginning of these symptoms in the patient coincided with her journey abroad (Egypt, Rhodes). First, the patient obtained ambulatory anti-fungal treatment (itraconazole, ketoconazole, nystatin) due to positive results of swabs for fungi: scalp - Penicillin, foot skin Candida gulliermondii, Microsporum gypseum, oral mucosa - Candida albicans. Multiple bacterial swabs were taken from the skin resulting in positive results for Staphylococcus epidermidis. Anti-histamine drugs and local emollients were also used with no improvement. At the same time, the patient was diagnosed with cirrhosis and started the treatment in the Gastroenterology Clinic (protective drugs). Diagnosis was confirmed by ultrasonography, computed tomography (CT) of abdomen, endoscopy of intestine and fine needle liver biopsy. In 2008, epidermal and point tests were negative. In 2009, due to increased complaints of the patient about the skin, the patient was admitted to the Dermatology Clinic of the Silesian Medical University in Katowice. The patient complained of her body itching, feeling of parasites crawling under her skin and in her eye. She also provided a jar containing pieces of wool and hairs ("worms" that leave her skin) (Figure 1). Despite efforts to explain her what the provided things are, she upheld her version. Additionally, she denied information that her skin itching may result from the liver disease. At admission, no fresh skin changes were observed. Single linear scars in the form of excoriations were seen on the trunk.

In additional laboratory tests, increased erythrocyte sedimentation rate (ESR) (14/22) was found, while full blood count, biochemical tests ( $\mathrm{Na}, \mathrm{K}, \mathrm{Cl}$, Fe, creatine, urea, glucose, bilirubin, CPK), protein levels with electrophoresis, coagulation parameters, general urine tests were normal. Alanine aminotransferase (AIAT), asparagine aminotransferase (AspAT) and $\gamma$-glutamyltransferase (GGTP) were slightly increased. Tumour markers, CEA and CA 125, were normal, HbS, HCV - negative. X-ray of the upper gastrointestinal tract, chest P-A, and ultrasonography of the abdomen were performed. No deviation from normal results was found. Mycological tests (scalp, foot skin, oral mucosa, anus area, vagina) - negative.

Ophthalmologic examination showed no deviation. Neurological examination indicated a psychosomatic character of the patient's complaints.

Psychological examination confirmed selected tactile hallucinations and suggested the need for observations for paraphrenic disturbances and a need for psychiatric examination. Psychiatric examination was focused on somatisation disturbances and indicated the need for further special psychiatric treatment. 
When in the Dermatology Clinic, symptomatic treatment with anti-histamine drugs and emollients was applied. The patient was referred for further diagnostic procedures and treatment in the mental health centre.

\section{Case 2}

Patient 57 years. For the past 3 years the woman was complaining about development of the skin changes such as pustules, papules and erosions on the arms, neck and décolleté. The skin changes were accompanied by itching, burning and pain. The patient denied irritating and provoking the skin changes. The patient takes care of her disabled son, as a single mother. She actively seeks assistance from social care institutions and has a habit of writing applications to different institutions (court, municipal office, police). First, she was treated in an outpatient clinic, then in her local Dermatology Department. The treatment involved local and general antibiotics, without any improvement of the local changes. Multiple bacteriological swabs were taken in the clinic with the result of Staphylococcus epidermidis. In 2008, the patient was diagnosed with irritable bowel disease. In 2009, she was admitted to the Dermatology Clinic of the Silesian Medical University in Katowice due to intensification of the skin changes. The admission was preceded by another document for the Hospital Manager containing the patient's demands. The woman complained about appearance of painful skin changes, which disintegrate without any inference. She also provided an envelope containing seeds, which were supposed to appear from the skin changes. The patient did not react to all attempts to explain the nature of the brought material and categorically denied her involvement in development of the skin changes.

At admission, the skin changes were localised on décolleté and lateral neck areas. They consisted of multiple, scattered, brown hyperpigmentations, with oozing erosions, erythema and scarring in the central part. The changes on the neck were of a linear character (Figure 2).

Additional tests: ESR (4/12), biochemical tests ( $\mathrm{Na}, \mathrm{K}$, $\mathrm{Cl}$, Fe, creatine, urea, glucose, bilirubin, AlAT, AspAT, GGTP and (PK), protein levels with electrophoresis, coagulation parameters, general urine tests were normal. Full blood count - leukopenia of a small degree. Tumour markers, CEA and CA 125, were normal, HbS, HCV - negative. X-ray of the upper gastrointestinal tract, chest P-A, and ultrasonography of the abdomen were performed. No deviation from normal results was found. Epidermal tests positive result for formalin. Mycological tests (décolleté, neck) - negative. Anti-nuclear antibodies ANA - indirect immunofluorescence (IF) in monkey's and guinea pig oesophagus and in HEp-2 cells - negative. Examination by an internist and haematologist - no deviations.

Psychological examination confirmed presence of a personality disorder (compensation personality). Psy-

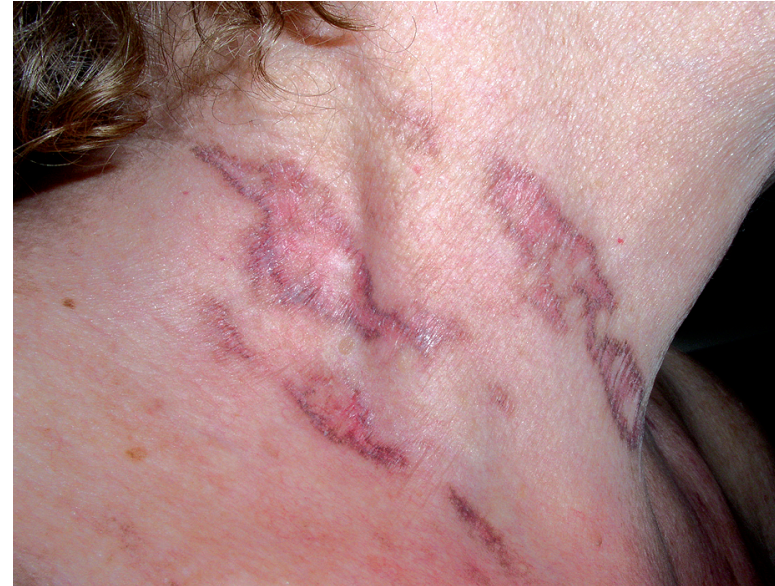

Figure 2. Patient 57 years - multiple, scattered, brown hyperpigmentations, with oozing erosions, erythema and scarring in the central part

chiatric examination suggested self-injuries and indicated the need for further special psychiatric treatment.

When in the Dermatology Clinic, symptomatic treatment with anti-histamine drugs and emollients was applied. The patient was referred for further diagnostic procedures and treatment in the mental health centre.

\section{Case 3}

Patient 56 years. First changes within the skin and subcutaneous tissue of both breasts were found about 30 years ago. The changes formed nodular infiltrates with a tendency to fibrosis and formation of white-yellow hard foci. First, Panniculitis non febrilis was diagnosed and since 1986 - Scleroderma profunda. Treatment included glucocorticosteroids, immunosuppression, procaine penicillin, penicillaminum, Piascledine (Avocado oleum, Soiae oleum), sulfones and antibiotics. Temporary exacerbations were characterised by intensification of local inflammatory changes with formation of small sores. In 1994, the patient was diagnosed with ventricular tachycardia (the patient is under cardiologist care). The woman has been undergoing control examinations in the Dermatology Clinic for 23 years. In 2008, she was admitted again due to exacerbation of the local condition. At admission, the changes involved the skin and subcutaneous tissue of both breasts and were of nodular-infiltrative character. The foci were irregular and showed deep, desk-like hardenings and were painful upon palpation. The nipples were surrounded by yellow foci with increased cohesiveness, surrounded by violet-pink aureoles. Oval or round scars and longitudinal skin fissures were found (Figure 3).

Additional tests: ESR: OB (24/36), full blood count, biochemical tests ( $\mathrm{Na}, \mathrm{K}, \mathrm{Cl}$, Fe, creatine, urea, glucose, bilirubin, AIAT, AspAT, GGTP and (PK), protein levels with electrophoresis, coagulation parameters, general urine tests were normal. Tumour markers CEA and CA 125 were normal, HbS, HCV - negative. X-ray of the upper gastroin- 


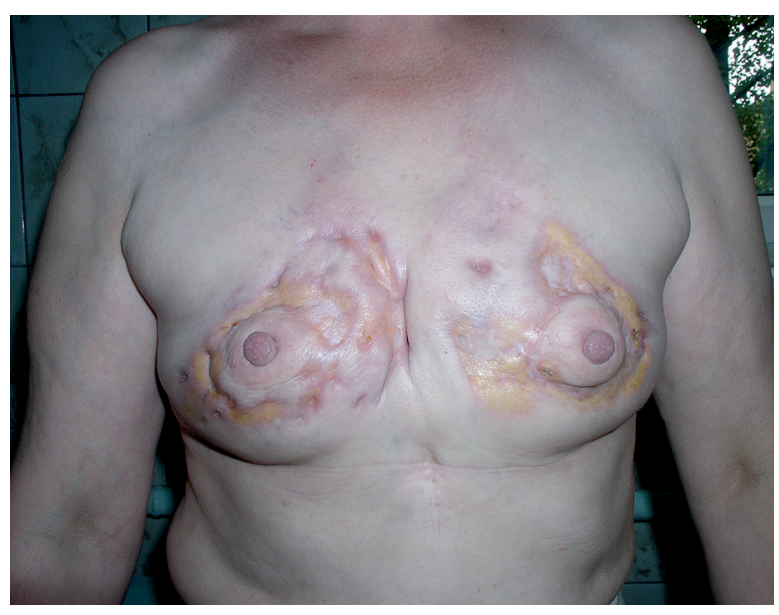

Figure 3. Patient 56 years - irregular and showed deep desklike hardenings with the nipples surrounded by yellow foci with increased cohesiveness, surrounded by violet-pink aureoles

testinal tract, chest P-A and comparative X-ray of hands, and ultrasonography of the abdomen were performed.

All tests' results were normal. Mammography - X-ray image indicates cystoid, fibrous and mastopathic changes with a component of Fibrosclerosis changes. Single adenofibromas, extensive sclerodermal changes of the skin of both breasts. Ultrasonography of breasts - the changes are localised within breasts, but their character and scope excludes a possibility of an ultrasonography diagnostic procedure. Two-contrast colonography - normal.

Magnetic resonance imaging (MRI) of both breasts presence of extensive, solid infiltrative changes involving the skin and subcutaneous tissue as well as the gland tissue at the depth of 11-13 mm, the changes also involve the skin between breasts. Visible tissue lesion in the left breast as in the examination. Under the lesion, signals are such as in another area of infiltration. The changes are evenly intensified without pathological intensifica-

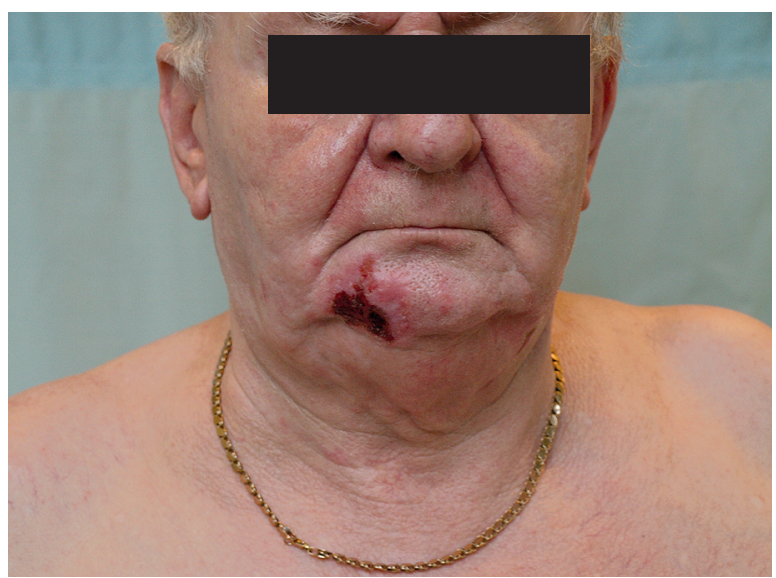

Figure 4. Patient 68 years - multiple, scattered, brown nodules and erosions covered with copious oozing serous excretion tion. The delayed sequence showed even intensification of tissues indicating that the process is active. Oesophagus passage - no significant delay in the passage of liquid contents through the oesophagus. Spirometry - no abnormalities in the respiratory tract. Computed tomography of the chest - lungs without focal densities. No increased lymph nodes within mediastinum. Scars seen within breasts, probably related with the basic disease.

Anti-nuclear antibodies ANA - IF in monkey's and guinea pig oesophagus and in HEp-2 cells - negative. IgG and IgM antibodies against Borrelia burgdorferi- negative.

Histopathology (1992) - multiple fibrosis and giant cells phagocytising lipids in the dermis (lipogranuloma cum fibrosi?), (lipogranulomatosis subcutanea? Rothman) and multiple enlarged lymphatic vessels. The patient did not agree for further histopathology. Mycological tests negative. Bacteriological tests - Staphylococcus epidermidis. Examination by the laryngologist, dentist and internist - no abnormalities.

Psychiatric examination - the patient did not agree at first, then, after she agreed, somatisations were found together with a possibility of dermatitis artefacta. When in the Dermatology Clinic, Piascledine (Avocado oleum, Soiae oleum) $2 \times 300 \mathrm{mg}$ and temporal treatment with antibiotics when small sores appeared was prescribed. The patient in a very good general state throughout the whole disease and was referred for further treatment in the mental health centre.

\section{Case 4}

Patient 68 years. The skin changes have been present for about 10 years in the form of papules, pustules, blisters, erosions and scars located on the face, trunk and arms. The appearance of changes was accompanied by itching, burning, pain and a feeling of "...woodworms or bacteria..." moving under the skin. The patient admitted the need for scratching to remove them. The changes irritated the skin and after removing them, the patient felt relief. He denied his involvement in the appearance of the primary changes. First, he was treated in the outpatient clinic, then in his local Dermatology Department. The therapy included local and general antibiotics without improvement of local changes. Multiple bacteriological swabs were taken in the outpatient clinic with the result of Staphylococcus epidermidis and enterobacter kobei (once). In 2007, the patient was diagnosed with diabetes type II. In 2009, he was admitted to the Dermatology Clinic of the Silesian Medical University in Katowice due to intensification of the skin changes.

At admission, the changes were localised on the chin, trunk and arms. They represented multiple, scattered, brown nodules and erosions covered with copious oozing serous excretion. Some of the erosions were covered with crusts. The arms showed a linear character of changes (Figure 4). 
Additional tests: ESR: OB (24/36), full blood count, biochemical tests ( $\mathrm{Na}, \mathrm{K}, \mathrm{Cl}$, Fe, creatine, urea, glucose, bilirubin, AIAT, AspAT, GGTP and (PK), protein levels with electrophoresis, coagulation parameters, general urine tests were normal. Tumour markers, CEA and CA 125, were normal. Slightly increased PSA. X-ray of the chest P-A, mandible and ultrasonography of the abdomen were performed. No abnormalities were found. Epidermal tests positive for nickel sulphate. Mycological tests (chin, trunk) - negative. Examination by the ophthalmologist, dentist, internist - no abnormalities. Surgical examination showed haemorrhoids II/III and prostatic enlargement.

Psychiatric examination confirmed neurotic and somatisation disturbances as well as parasitophobia and self-injuries. The treatment was extended by doxepinum $3 \times 10 \mathrm{mg}$ and further psychiatric diagnostic procedures and treatment was recommended.

When in the Dermatology Clinic, treatment with antihistamine drugs, local antibiotics and UVA light was applied. The patient was referred for further diagnostic procedures and treatment in the mental health centre.

\section{Discussion}

Diagnosis of an induced dermatosis should be based upon clinical suspicion and exclusion of the skin diseases $[1,7,8]$. Changes characteristic of dermatitis artefacta frequently well imitate "real" dermatological diseases at the beginning. However, over time, the clinical picture and results of additional tests do not represent a logical wholeness. The patient's state does not reflect the tests' results, it does not improve after the applied treatment, either. In most cases, the changes are localised in the body areas that are accessible to hands. Such situation was also found in all patients described here. The changes: papules, pustules, blisters, erosions and scars are also typical. Sometimes, the shape of the skin changes may reflect the shape of the object used for self-inflictions. The linear character, such as the one found in patient 2 and 4 , are also very common.

Mercan et al. [2] considers the factors, which play a role in development of such behaviours in patients. Authors divide them into three categories: stress factors (environmental, subjective feelings, biological response to stress), certain dermatological aetiology, which leads to exacerbation of a small skin problem and the primary psychiatric aetiology. The aetiological factors frequently co-exist in patients.

In patient 3, even a detailed psychological analysis did not allow us to define a clear picture of disturbances, which led to a very advanced self-injury. Self-inflicted injuries to breasts in women have already been the subject of research. Rodriquez-Pichardo et al. [9] conducted a retrospective assessment of this phenomenon in 1976-2006 within one dermatological department. Changes of this type were found in 27 patients with the mean age of 34.33 years. The most common form of changes is self-inflicted erosions and sores (in 6 patients, they were developed by burns). Other changes were: blisters, hematomas, and change of contact dermatitis type. In 17 patients, the changes were localised on breasts, face, arms and stomach.

Korabel et al. [10] estimate that tendencies to conscious or unconscious self-injuries are found even in $30-60 \%$ of the patients of dermatological departments, while depression symptoms are found in 30\% of patients primarily visiting a dermatologist [11].

Jafferany [3] noticed that patients with psychodermatoses significantly more frequently are characterised by psychotic personality features, tendency to anger behaviour and depressive behaviours. The work by Bria and Rinaldi [12] tested the level of anger and depression in patients with self-injuries. These patients were also characterised by a significantly higher frequency of traumatic events in their history. In our work, patient 2 had a difficult life situation, being a single mother. In others, no problems with the living situation were found.

Harth et al. [13] defined a set of symptoms presented by psychodermatologic patients (itching, pain, paresthesia, parasitophobia, hypochondria), as so called "nihilodermia". Patients with this diagnosis represent a diagnostically difficult group and it usually takes a long time to diagnose psychodermatosis. Poot et al. [14], in agreement with ESDaP (European Society for Dermatology and Psychiatry), described features of alexithymia in dermatological patients. Alexithymia means a need to be understood by others on the basis of one's appearance, including self-inflicted skin changes.

Apart from subjective sensations, such as the presence of "worms" in and under the skin, patients 1, 2, 4 most of all experienced itching.

Misery et al. [15] proposed in 2007 diagnostic criteria of itching according to the French Psychodermatologic Group (FPDG). They defined three criteria of compulsive behaviours: general itching without primary changes on the skin, the itching lasts for more than 6 weeks, somatic reasons for this ailment were excluded. Additional criteria are: chronological relation between life situations with psychological complications and itching, relation between itching intensity and stress situations, increased itching at night and during rest and lack of activities, coexistent other psychic disorders, improvement after psychotherapy and psychotropic drugs.

Psychogenic itching involves particular body parts: face, head and genital area. The skin changes are also located in these areas with no other symptoms [16]. In our patients with a parasitophobia, the changes were more scattered.

In two cases described above, diagnostic procedures involved histopathological testing of the skin biopsies. In the patient with self-injuries to breasts, these biopsies were taken many times, due to the long time of disorder 
and suspected autoimmunity background (scleroderma?). Kwon et al. [17] underline the necessity of such tests, due to frequently an unclear clinical picture of dermatitis artefacta. Authors presented a patient with recurring sores, in whom no etiological agent had been found for many years. The histological test made it possible to show the presence of giant cells reaction around the external body. The factors were cellulose factors, placed by the patient herself under her skin.

The therapy of psychodermatologic diseases is difficult and it always requires a close co-operation between the dermatologist, psychologist and psychiatrist [18]. Doctors from these groups comprise a so called holistic group managing the patient [19]. Dermatological treatment is exclusively symptomatic. In most cases, sores heal under occlusive dressing, when the patient has no access to his skin. Anti-histamine drugs are not always efficient, due to the psychogenic background of itching and due to other subjective sensations of the patient. In the case of bacterial, fungal or viral superinfections, it is necessary to include a specific local or general treatment. Psychiatric treatment is the most important part of the therapy, frequently leading to the total healing of skin changes [19]. Therapy may have a pharmacological or non-pharmacological character. The pharmacological means belong to three main groups: antidepressants (doxepinum, clomipramine, fluoxetine), tranquilisers (benzodiazepines) and anti-psychotic (pimozide, fluspirilene, haloperidol) [1, 6]. However, one should remember about multiple skin adverse effects of these drugs: photoallergies, dark skin pigmentation, contact dermatitis, urticaria, post-drug lupus, erythema, blister changes, erythema multiforme, TEN, vasculitis, sores (lit), acne-like and psoriasis-like changes, leuconychia, hair loss etc. These can additionally have a negative influence upon the state of a patient with psychodermatosis [3]. Non-pharmacological methods include multiple forms of therapies used in special centres, such as psychotherapy, hypnosis, relaxations, behavioural therapy, meditation, stress control and others [3]. The aim of a psychotherapist is to reduce subjective feelings of a patient itching and related urge to scratch, also decrease negative emotions, such as anger, sadness [6]. Fried [6] believe that non-pharmacological methods should be used for a long time, even as a life-time means, due to a chronic character of psychodermatologic disorders and a high percentage of recurrence after the therapy end. Non-pharmacological treatment can be led by a psychologist, psychiatrist, behavioural therapist, and also by specially trained social workers.

We have presented four cases of patients with invoked dermatoses, in which diagnosis of self-injuries and parasite phobias was preceded by long-term and detailed diagnostic procedures for classical dermatological disorders.

One should always remember about dermatitis artefacta in patients, in whom there is no positive response to treatment, and the clinical picture as well as results of additional tests comprise no logical whole picture.

\section{References}

1. Braun-Falco O, Plewig G, Wolff HH, Burgdorf WHC. Itching, psychiatric disorders, neurologic disorders. In: Dermatology. Braun-Falco O, Plewig G, Wolff HH, Burgdorf WHC. Czelej, Lublin 2002; 931-52.

2. Mercan S, Kivanç Altunay I. Psychodermatology: a collaboration between psychiatry and dermatology. Turk Psikiyatri Derg 2006; 17: 305-13.

3. Jafferany M. Psychodermatology: a guide to understanding common psychocutaneous disorders. Prim Care Companion J Clin Psychiatry 2007; 9: 203-13.

4. Steuden S, Janowski K. Psychodermatologic disorders. Przegl Dermatol 2002; 3: 175-83.

5. Stankiewicz-Choroszucha BL, Wawrzyniak ZM, Lipiec A, et al. Consequences of smoke inhalation in the 'Epidemiology of Allergic Diseases in Poland' project (ECAP). Ann Agric Environ Med 2011; 18: 420-8.

6 . Fried RG. Nonpharmacologic treatments in psychodermatology. Dermatol Clin 2002; 20: 177-85.

7. Buljan D, Buljan M, Situm M. Psychodermatology: a brief review for clinicians. Psychiatr Danub 2005; 17: 76-83.

8. Kieć-Swierczyńska M, Dudek B, Krecisz B, et al. The role of psychological factors and psychiatric disorders in skin diseases. Med Pr 2006; 57: 551-5.

9. Rodríguez-Pichardo A, Hoffner MV, García-Bravo B, et al. Dermatitis artefacta of the breast: a retrospective analysis of 27 patients (1976-2006). J Eur Acad Dermatol Venereol 2009; 20: $345-76$.

10. Korabel H, Dudek D, Jaworek A, et al. Psychodermatology: psychological and psychiatrical aspects of dermatology. Przegl Lek 2008; 65: 244-8.

11. Filaković P, Petek A, Koić O, et al. Comorbidity of depressive and dermatologic disorders - therapeutic aspects. Psychiatr Danub 2009; 21: 401-10.

12. Bria P, Rinaldi L. The psychic skin: principles and prospectives of intervention in psychodermatology. Clin Ter 1999; 150: 287-93.

13. Harth W, Hermes B, Seikowski K, et al. Nihilodermia in psychodermatology. Hautarzt 2007; 58: 427-34.

14. Poot F, Sampogna F, Onnis L. Basic knowledge in psychodermatology. J Eur Acad Dermatol Venereol 2007; 21: 227-34.

15. Misery L, Alexandre S, Dutray S, et al. Functional itch disorder or psychogenic pruritus: suggested diagnosis criteria from the French psychodermatology group. Acta Derm Venereol 2007; 87: 341-4.

16. Lotti T, Buggiani G, Prignano F. Prurigo nodularis and lichen simplex chronicus. Dermatol Ther 2008; 21: 42-6.

17. Kwon EJ, Dans M, Koblenzer CS, et al. Dermatitis artefacta. J Cutan Med Surg 2006; 10: 108-13.

18. Locala JA. Current concepts in psychodermatology. Curr Psychiatry Rep 2009; 11: 211-8.

19. Tordeurs D, Poot F, Janne P, et al. Psychological approach to different skin diseases: life events and tendency to complain. Ann Dermatol Venereol 2001; 128: 21-4. 